Radial and Nonradial Pulsations as Probes of Stellar Physics

ASP Conference Series, Vol. 259, 2002

C. Aerts, T.R. Bedding, \& J. Christensen-Dalsgaard, eds.

\title{
Seismic Constraint on the Plausible Explanations of the Metal Rich Feature of Planetary System Stars
}

\author{
H. Shibahashi, S. Watanabe \\ Department of Astronomy, University of Tokyo, Japan
}

\begin{abstract}
From spectroscopic observations showing that the parent stars of planetary systems are metal rich, it is suspected that this feature results from the accompanying planetary system. With the rich helioseismic data so far obtained, we determine the metal abundance distribution in the solar interior with respect to the distance from the center. This result gives constraints on the possible explanations about the metal rich feature of the stars accompanied by a planetary system.
\end{abstract}

\section{Introduction}

One of the recent exciting topics in astrophysics is the discovery of extra-solar planetary systems. After the first monumental discovery, more than 70 systems have so far been found. Stimulated by this rush of extra-solar planets hunting, various kinds of investigation have been carried out. One of them is careful spectroscopic observation of the parent stars of those systems. The systematic observations surprisingly showed that most of those stars are substantially metal rich compared with the Sun (Gonzalez et al., 2001 and references therein). It should be noted that the Sun, which is the prototype of a planetary system star, is also relatively metal rich compared with its nearby stars (e.g., Snow \& Witt, 1996). From these facts, one might suspect that the metal rich feature of the stars associated with a planetary system results from the presence of the planetary system. One of the plausible explanations is that if the proto-stellar gas is initially rich in the heavy elements, this results in formation of a planetary system at the star formation phase and hence the parent star is rich in heavy elements (scenario A). Another possible explanation is that, assuming that planets are formed by accumulation of heavy elements in normal abundance proto-stellar gas, the envelope of the parent star, which has normal metal abundance at the initial phase, is contaminated by accretion of the remaining metal rich gas components (scenario $B$ ).

According to scenario (A), the metal abundance distribution in the parent star is supposed to be uniform or to be slightly condensed in the deeper region due to the gravitational settling. On the other hand, in the case of the scenario (B), the metal abundance is likely to be rich in the outer envelope. Hence, if we could see how the heavy elements are distributed in the parent star, we would be able to discriminate between these two scenarios. Though the interior of distant stars is invisible, the development of helioseismology provides us new eyes to see inside the interior of the Sun (Deubner et al., 1998). 


\section{How can the $Z$-profile in the Sun be determined ?}

The reason why the solar internal structure can be determined with helioseismic constraints is explained as follows. If we write symbolically the basic equations describing the linear, adiabatic oscillations of the Sun in terms of an operator $\mathcal{L}$,

$$
\sigma^{2} \boldsymbol{\xi}=\mathcal{L}(\boldsymbol{\xi})
$$

where $\sigma$ and $\boldsymbol{\xi}$ denote the eigenfrequency and the displacement eigenfunction, respectively, the operator $\mathcal{L}$ is expressed in terms of $\boldsymbol{\xi}$ and the two physical quantities of the equilibrium structure of the Sun; - i.e., the sound speed $c(r)$ and the density $\rho(r)$. Introducing a reasonable model of the Sun and applying the variational principle to the above equation, we deduce the difference in $c(r)$ and $\rho(r)$ between the model and the real Sun, and eventually $c(r)$ and $\rho(r)$ of the real Sun. Indeed, helioseismology has been successful in determining precisely $c(r)$ and $\rho(r)$ in the Sun. The problem is how to deduce the $Z$-profile from such seismic information.

The basic equations governing the radiative core of the Sun are the continuity equation, the hydrostatic equation, the energy equation, and the energy transfer equation. The dependent variables appearing in the left-hand-side of these equations are $M_{r}, P, T$, and $L_{r}$. In the right-hand-side, however, besides these variables, $\rho, \varepsilon$, and $\kappa$ are appearing. Hence, in addition to these differential equations, we need auxiliary equations; - that is, the equation of state, the equations for the opacity, and the nuclear reaction rates: $\rho=\rho\left(P, T, X_{i}\right)$, $\kappa=\kappa\left(P, T, X_{i}\right)$, and $\varepsilon=\varepsilon\left(P, T, X_{i}\right)$. If we distinguish only the hydrogen and helium separately as $X$ and $Y$, respectively, and treat all the other elements collectively as heavy elements $Z$, then the sound speed and the density can be regarded as functions of the chemical composition, $X$ and $Z$, and any two other thermodynamical quantities such as $P$ and $T ; c=c(P, T, X, Z)$ and $\rho=\rho(P, T, X, Z)$. It should be reminded here that the sound speed profile and the density profile in the solar interior have been determined from helioseismology. Hence the hydrogen abundance $X$ and the heavy elements abundance $Z$ at a given $r$ are related to the pressure, the temperature, the sound speed, and the density; $X=X\left(P, T, c_{\text {obs }}, \rho_{\text {obs }}\right)$ and $Z=Z\left(P, T, c_{\mathrm{obs}}, \rho_{\mathrm{obs}}\right)$, where $c_{\mathrm{obs}}(r)$ and $\rho_{\text {obs }}(r)$ denote the seismically determined sound speed profile and the density profile, respectively. The opacity and the nuclear reaction rate are, in turn, given in terms of $\left(P, T, c_{\mathrm{obs}}, \rho_{\mathrm{obs}}\right)$. Thus all the variables appearing in the righthand-side of the basic equations can be expressed in terms of the dependent variables in the left-hand side, and hence these equations are solvable. Note that in this way we obtain directly a present solar model without following the evolution of the Sun. Note also that we do not need to make assumptions about the chemical composition profiles in the Sun, but obtain the hydrogen- and the helium-profiles as part of the solutions of the fourth-order differential equations of stellar structure (Watanabe \& Shibahashi, 2001).

The boundary conditions at the center are trivial: $M_{r}=0$ and $L_{r}=0$ at the center. We adopt the seismically determined depth of the convection zone, $r=r_{\text {conv }}$, and set the outer boundary conditions there; the temperature gradient must be the adiabatic one, and $L_{r}=L_{\odot}$. This means that we do not need to care about the convective heat transport, which has theoretical 
uncertainties (Takata \& Shibahashi, 1998). Moreover, chemical homogeneity in the convection zone requires that the abundance ratio of metal to hydrogen, $Z / X$, at the base of the convection zone should be identical with the value at the photosphere, $(Z / X)_{\text {surf }}$, which is determined spectroscopically.

\section{How to determine seismically the $Z$-profile in the Sun ?}

It is in practice hard to determine the $Z$-profile directly in the way described in the previous section, since the dependence of the equation of state upon $Z$ is so weak. Therefore, we devise a more practical method of solving the basic equations for the stellar structure together with a constraint of $c_{\mathrm{obs}}(r)$ and $\rho_{\text {obs }}(r)$. In this method, we construct seismic solar models with a constraint of the helioseismically determined sound speed profile by assuming various $Z$ profiles, and we search among them for the model of which the density profile fits best the helioseismically determined density profile. In doing so, we expand the $Z$-profile by $Z(r)=Z_{0}+\sum_{i} a_{i} \delta Z_{i}$, where $Z_{0}$ is a certain constant $(=0.0171)$, $\delta Z_{i}$ is a piecewise constant function, $\delta Z_{i}=0.001$ only for $r_{i-1} \leq r \leq r_{i}$ and 0 otherwise, and $\left\{a_{i}\right\}$ are the expansion coefficients. As a linear response, the density profile is given by $\rho(r)=\rho_{0}(r)+\sum_{i} a_{i} \delta \rho_{i}(r)$, where $\rho_{0}(r)+\delta \rho_{i}(r)$ is the linear response function to $Z=Z_{0}+\delta Z_{i}$. Our goal is to find among various seismic solar models the model which minimizes the following integral:

$$
F=\int_{0}^{r_{\mathrm{conv}}}\left(\frac{\rho_{0}(r)+\sum_{i} a_{i} \delta \rho_{i}(r)-\rho_{\mathrm{obs}}(r)}{\sigma_{\rho(\mathrm{obs})}(r)}\right)^{2} \frac{d r}{R_{\odot}},
$$

where $\sigma_{\rho \text { (obs) }}(r)$ is the error of the helioseismically determined density. Here the distance from the center, $r$, is divided into 6 segments with a width $\Delta r / R_{\odot}=0.1$ but for the innermost segment $r_{0} / R_{\odot}=0.0$ and $r_{1} / R_{\odot}=0.20$. We restrict ourselves to supposing that $\left\{a_{i}\right\}$ are integers and that $0.010 \leq Z(r) \leq 0.025$ for any $r_{i}$. Furthermore, we require that the $Z$-profile is a fairly smooth function of $r ;$ - that is, $\left|a_{i+1}-a_{i}\right| \leq 4$.

\section{Results}

As for the helioseismically determined profiles of $c_{\text {obs }}(r)$ and $\rho_{\text {obs }}(r)$, we have adopted those obtained by Basu (1988) using the first 144-days MDI data. Fig. 1 (left panel) shows the best-fit $Z$-profile obtained in this way. However, the function $F$ is not so severely sensitive to the $Z$-profile, and even substantially different forms of $Z(r)$ sometimes lead to similar values of $F$. In this situation it is hard to determine uniquely the functional form of $Z(r)$. Hence we average all the $Z$-profiles leading to $F \leq 2 F_{\min }$, where $F_{\min }(\simeq 0.36)$ is the minimum value of $F$ corresponding to the profile shown in Fig. 1, and regard the averaged profile as the most likely profile. The averaged $Z$-profile is shown in Fig. 1 (right panel) by the solid line, and the range of variance of all the $Z$-profiles leading to $F \leq F_{\min }$ is shown by dashed lines.

The averaged profile is a monotonic function. This seems more favorable for scenario (A) than for scenario (B). However, the age of the Sun is believed to be 

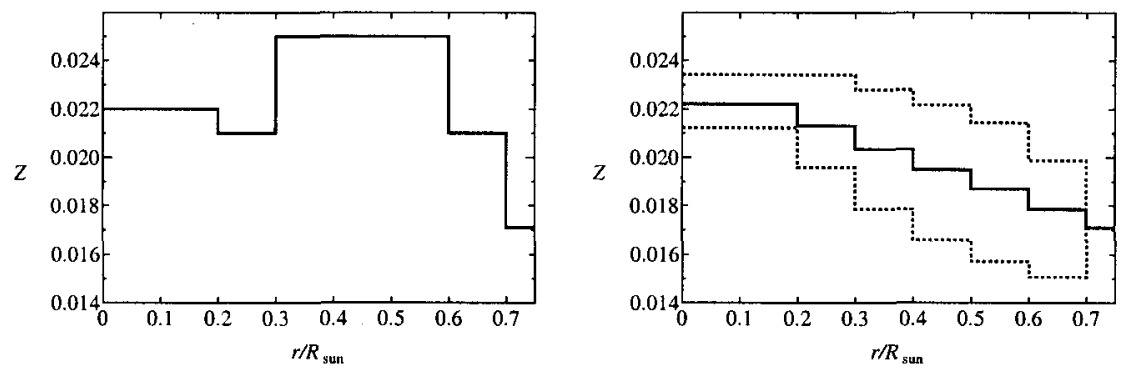

Figure 1. Left (a): The best-fit profile of $Z(r)$, which minimizes $F$ and satisfies the bias conditions (see the text). Right (b): The averaged profile and the range of variance of $Z(r)$ satisfying $F \leq 2 F_{\min }$, where $F_{\min }$ is the value of $F$ corresponding to the $Z$-profile shown in the left panel.

longer than the timescale of the material diffusion in the Sun, and so scenario (B) is not completely ruled out at this stage. Before reaching a definite conclusion, we will have to consider time evolution of the metal abundance profile with various initial conditions and will have to look for the best evolution scenario for explanation of the helioseismically deduced $Z$-profile of the present days Sun.

\section{References}

Basu, S. 1998, MNRAS, 298, 719

Deubner, F.-L., et al. (eds.) 1998, New eyes to see inside the Sun and stars, (Kluwer Academic Publishers)

Gonzalez, G., Laws, C., Tyagi, S., \& Reddy, B.E. 2001, AJ, 121, 432

Snow, T.P. \& Witt, A.N. 1996, ApJ, 468, L65

Takata, M. \& Shibahashi, H. 1998, ApJ, 504, 1035

Watanabe, S. \& Shibahashi, H. 2001, PASJ, submitted

\section{Discussion}

I. Roxburgh : I am surprised that you can determine the variation of $Z$ using a seismic model. The inversions in practice only give one function in the interior since the second independent variable is effectively the adiabatic exponent $\Gamma_{1}$, which is very close to $5 / 3$ in the bulk of the interior. It is only $\Gamma_{1}-5 / 3$ that contains information and this is not effectively determined by seismic inversion, at least not to an accuracy that can be used to constrain the composition.

H. Shibahashi : Helioseismic inversions provide us two independent variables. As far as we adopt the sound speed and the density as these variables, the resultant $\Gamma_{1}$, which is obtained as a solution of the seismic solar model, deviate meaningfully from $5 / 3$, as in the case of the evolutionary models. 